\title{
VALEUR NUTRITIVE D'UN RÉGIME A BASE DE CÉRÉALES, POMMES DE TERRE ET LAIT ÉCRÉMÉ POUR L'ALIMENTATION DU POUSSIN
}

\author{
PAR \\ C. CALET et P. DELPECH \\ Station de Recherches avicoles, C. N. R. Z., Jouy-en-Josas (S.-et-O.).
}

\section{INTRODUCTION}

Le but de cette expérience est d'établir un plan d'alimentation à base de céréales, lait écrémé et pommes de terre, produits faciles à obtenir sur la plupart des exploitations agricoles. De tels essais ont déjà été proposés, chez la poule pondeuse ('Temperton et Dudrey (57). Schlamb et BRYANT (49)). Il était intéressant de tenter de transposer à l'élevage du poussin les effets heureux reconnus au lait et aux pommes de terre pour les pondenses et les jeunes animaux (LEROY et col. (32 et 33), STEVENS (54)).

A la ferme, le lait écrémé de vache est un précietux aliment (CASTLE et col. (8)). Calculé par rapport à la matière sèche, il est riche en protéines $(37 \%)$ qui sont d'excellentes qualités ('TERROINE (58)). Ces protéines sont d'ailleurs identiques à celles du lait frais (Trémolrì̀res et col. (6o)).

D'une part, chez le jeune mammifère qui le boit, l'azote du lait présente un coefficient d'utilisation digestive très élevé (RANDorn et col. (43)). D'autre part, à la suite de travaux sur le Rat blanc, BLOck et Mr'TCHFLL (4) ont établi une classification des différents protides alimentaires quant à leur efficacité pour la croissance. D'après ces valeurs publiées en I946, les protéines du lait se classent en second, juste après 1'œuf, tant pour leur valeur biologique qui est égale à 90 (cette valeur est de 96 pour l'œuf), que pour leur coefficient d'utilisation pratique, 86 contre 93 pour l'œuf.

La cause de cette supériorité réside en grande partie dans la richesse en lysine des protéines du lait.

I a comparaison des acides aminés du lait et des céréales par exemple, met parfaitement en évidence les avantages protéiques du lait. Alors que les protéines des céréales ne contiennent que 2,7 p. roo de lysine en moyenne (Bourdet (6)), celles du lait en contiennent 7,5 p. Ioo (MitCHEL,L, et BLOCK (38)). 
Il semble donc avantageux sous ce rapport de supplémenter les céréales par le lait écrémé. De fait, MULLER et col. (4I) chez le porc d'une part, et HAR'T (I9) chez le rat d'autre part, observent les meilleures intensités de croissance avec des régimes constitués de $50 \mathrm{p}$. Ioo de lait et $50 \mathrm{p}$. Ioo de céréales.

D'autres auteurs ont également vanté les qualités des protéines du lait. Chez les volailles, OTT et col. (42) montrent que la lactalbumine corrige parfaitement les déficiences en acides aminés d'une ration classique pour poussin, mieux que ne le font les farines de viande. Cette supériorité s'explique par la richesse des protéines du lait à la fois en lysine, en tryptophane et en méthionine (SpISNI (53)).

I,es qualités du lait ne se limitent pas au seul domaine protéique. $I_{4}$ lait écrémé est en effet une source appréciée de sels minéraux et de vitamines.

Les quantités de calcium du lait sont très importantes. Elles représentent environ I p. Ioo de la matière sèche (ABDiRHALDEN (I), BUNGE et col. $(6$ bis $)$ ). De plus, la valeur très élevée du rapport phospho-calcique de cet aliment - I,3 (Rochaix et Tapernoux (46)) - contraste avec celle des éléments végétaux, ce qui présage, là encore, une combinaison alimentaire heureuse du lait et des céréales. Enfin, une grande partie du phosphore du lait est sous forme organique. On sait, par exemple que les $3 / 4$ de la caséine sont phosphorylés ('TREMOLIÈRES (60)). Il convient d'insister sur ce résultat car le phosphore lié aux protéines animales est particulièrement bien assimilé (RICHET (45)).

I.e lait est donc une source intéressante de calcium. I1 a ell plus l'arantage de faciliter son utilisation. Duncan (I4) d'tune part et Fournier et col. (I6) d'autre part, insistent sur la faculté que possède le lactose du lait à favoriser l'absorption et la rétention calciques. Ils remarquent aussi - comme nous le verrons plus loin - que le lactose doit être utilisé à une dose convenable et non excessive pour que ses bons effets soient observés.

Enfin, l'apport d'oligo-éléments minéraux par le lait n'est pas à dédaigner, sauf en ce qui concerne le fer (SLmoneT (52)).

Du point de vue vitaminique, il faut souligner que le lait écrémé est dépourvu de vitamines $\mathrm{A}$ et $\mathrm{D}$. I Les vitamines $\mathrm{B}$, au contraire, $\mathrm{y}$ sont bien représentées. Par exemple, le lait occupe la $3^{\mathrm{e}}$ place dans la hiérarchie des aliments humains après le pain et la viande de porc, en ce qui concerne sa teneur en vitamine BI (LANE et col. (28)). Toutes les autres vitamines $\mathrm{B}$ ont été dosées dans le lait en quantités non négligeables (Kon et col. (26-27), Hassinen et col. (20), DeBrit (II)).

I1 faut cependant signaler les variations importantes de la teneur vitaminique des laits sous l'influence de nombreux facteurs. C'est le cas en particulier de la Thiamine et de la Riboflavine, dont le taux dépend 
de la saison, du stade de lactation, etc. (Kon (25)). 'Toutefois, en dépit de ces variations, le lait reste encore une très bonne source de Riboflavine, puisque $6 \mathrm{p}$. Ioo de lait écrémé sec dans le régime en apportent des quantités suffisantes pour supplémenter un régime classique chez le poussin (BLACK et col. (3)).

Au cours de ces dernières années de nouvelles vitamines et facteurs de croissance ont été décrits dans le lait écrémé. C'est le cas par exemple de la vitamine BI2 qui s'y trouve largement représentée (I,IL,IE et col. (35)).

On a trouvé également en I94I une vertu particulière du lait écrémé pour la croissance du poulet, qui ne peut être reproduite que par les farines de viande et de poisson (Dickens et col. (I3)). Ce ou ces principes font l'objet, à l'heure actuelle, d'études très nombreuses et sont groupés sous le vocable "facteurs indéterminés du lait écrémé ou du petit lait " (MengF et col. (36), Camp et col. (7)). Ils seraient liés aux protéines du lait et non au lactose (ATKINSON et col. (2)).

Pour toutes ces raisons, l'éleveur trouve dans le lait écrémé qu'il produit sur place une source inestimable d'éléments nutritionnels. Ceci a été bien compris dans le cas de l'élevage du porc, mais chez le poulet, rares ont été jusqu'à ce jour les tentatives d'emploi du lait écrémé comme boisson dès le premier âge.

C'est qu'en effet l'usage alimentaire du lait n'est pas sans danger pour les jeunes animanx. Jacguor et Trimbach (24) observent des cas d'intolérance chez des animaux nourris au lait. SHAw $_{5} \mathrm{5}$ I) rapporte que des poussins meurent au bout de 3 jours s'ils reçoivent après l'éclosion, du lait comme seul aliment. Ils présentent alors une inflammation des parois gastro-intestinales.

Ces résultats ont été souvent confirmés chez d'autres espèces de volailles ( $\$ \operatorname{cotT}(50)$ ) et de nombreux auteurs ont reconnt au lait la propriété de favoriser les diarrhées. Pour eux le lactose en serait le responsable. Lorsque le poussin ingère plus de 2 grammes de lactose, les troubles digestifs apparaissent (Hamition et col. (I8)). Ces troubles peuvent se compliquer de déformation des doigts, de cataracte et même de convulsions épileptiques, lorsque les doses administrées sont trop fortes (RUTTER (48)).

Enfin, les dernières critiques d'ordre nutritionnel que l'on peut faire au lait écrémé tiennent à son encombrement et à son faible pouvoir énergétique. D’une part, le lait écrémé est un aliment très dilué. Il faut donc en boire beaucoup pour ingérer une quantité suffisante d'éléments nutritifs. D'autre part, l'écrémage du lait lui soustrait la plus grande partie de ses lipides et provoque une chute extrêmement importante de son taux calorique.

Il en résulte que le lait écrémé est relativement trop riche en protéines 
en regard de sa teneur peu élevée en principes énergétiques. C'est ce déséquilibre entre les protéines et l'énergie de cet aliment qui est la cause de déboires quand par exemple, il est employé seul dans l'alimentation du veau.

D'un autre côté de nombreux auteurs insistent sur l'aspect sanitaire du problème. Il arrive en effet que l'ingestion de lait entraîne des troubles digestifs qui résultent de contaminations de celui-ci ou de la malpropreté des récipients qui servent à sa collecte (IAUCHÈrE (I5)). Il est bien certain que le lait est un milieu de choix pour la culture de tous les microorganismes et qu'il demeure un aliment difficile à manipuler.

Compte tenu de ces données, nous nous sommes efforcés :

I $^{\circ}$ D'étudier un régime qui permette de combler les déficits d'énergie et de vitamines liposolubles du lait écrémé en utilisant des céréales, des pommes de terre, et éventuellement un aliment de complément.

$2^{\circ}$ De définir quelles quantités de lait écrémé peuvent être bues sans danger par le poussin et quelles précautions d'hygiène il est nécessaire de prendre pour l'utiliser dans la pratique.

Sous un autre aspect du problème, nous avons déterminé les conditions d'emploi des pommes de terre dans l'alimentation du poussin.

Ce tubercule est largement utilisé dans l'élevage des jeunes animaux. LEROY et ses collaborateurs (33) ont résumé les avantages de la pomme de terre comme aliment et ont précisé la manière de l'utiliser pour l'alimentation des porcins, bovins et ovins.

Les protéines de la pomme de terre sont constituées pour la plupart de tubérine dont la valeur biologique est très élevée ('TERroins, (58).) Jacouo' et Armand (23) déterminent l'efficacité des protides de la pomme de terre et trouvent une valeur comparable à celle de la caséine. La teneur en protéine de la pomme de terre est très faible $(2, \mathrm{I}$ p. Ioo de matière humide ou encore 8,4 de la matière sèche), mais il faut retenir que ces protéines sont d'excellente qualité - en particulier très riches en lysine (CHrck et col. (Io)) - et que leur teneur en acides aminés est relativement constante, quelles que soient les variétés (MULDER (40)).

D'un autre côté, les pommes de terre sont riches en phosphore (RANDorn et CAUSERE'T (44)), ce qui contraste avec leur pauvreté en calcium (LEROY et col. (33)). L'adjonction de lait aux pommes de terre est donc une opération avantageuse puisque le rapport phospho-calcique final s'en trouve amélioré.

Signalons enfin la richesse de ce tubercule en vitamine C (RANDors et col. (44), RoINE et col. (47)), dont une certaine partie est perdue lors de la cuisson. A ce sujet tous les auteurs ne sont pas d'accord sur l'importance des pertes de vitamine $\mathrm{C}$ selon le mode de cuisson. Certains pensent que l'autoclavage ne modifie pas le taux d'acide ascorbique mais 
diminue le taux d'acide déhydro-ascorbique (LESCHSENRING et col. (3I)). Ces auteurs trouvent le même résultat si la pomme de terre est cuite au four. L'ébullition, au contraire, provoque une solubilisation de la vitamine qui s'accompagne d'une élévation très marquée de la teneur en acide ascorbique de la pomme de terre soumise à ce traitement (LEICHSENRING et col. (30)). Par contre, dans les mêmes conditions Szczylowa et col. (55) aboutissent à la conclusion opposée puisqu'ils enregistrent tune perte de 40 à $50 \mathrm{p}$. Ioo de cette vitamine.

Ces données sont intéressantes à connaître car les pommes de terre doivent en effet être cuites pour être parfaitement digestibles. Alors que l'amidon cru de blé ou de riz a un coefficient d'utilisation digestive de 98 p. Ioo à la fois chez la souris (Denusses et Terrier (I2)) et chez le coq (Miromoto et col. (39)), la fécule de pomme de terre non chauffée est digestible à 40 p. Ioo.

Pour être utilisée, cette fécule doit être cuite (Chen et WANG (9)), ou broyée très finement ( $\mathrm{I}_{\text {EVY }}$ et JACQUOT (34)). Il faut en effet que la couche externe du grain d'amidon soit altérée (BooHer et col. (5)). Dans ces conditions les pommes de terre sont bien tolérées par le poulet quand il en reçoit $15 \mathrm{p}$. Ioo de son régime (MCMILI,AN et col. (37)). Chez le canard, Heuser et col. (2I) estiment que 1'on peut remplacer 30 p. Ioo de maïs du régime par de la farine de pommes de terre sans remarquer de modification de la croissance ni de l'indice de consommation.

Toutefois un excès de pommes de terre dans la ration est préjudiciable et provoque des croissances anormales (TEMPERTON et col. (56)) ou encore entraîne une élévation de l'indice de consommation et pousse les animaux à faire de la graisse ( $\mathrm{I}_{\mathrm{EH} \text { HAN }}(29)$ ). Dans le même ordre d'idées GERRY et col. (I7) reprochent aux sous-produits de la pomme de terre de ralentir la croissance et de réduire l'efficacité de la ration. Toutefois, ces auteurs remarquent que cette pratique aboutit à un abaissement notable du prix de revient du kilo de poulet. HöE et SANDviK (22) rapportent une expérience sur poules pondeuses où les pommes de terre cuites ( 350 grammes par jour) étaient distribuées avec un mélange de lait écrémé sec et de grains entiers. Bien que les résultats obtenus soient très légèrement inférieurs à ceux du témoin, il n'en reste pas moins vrai qu'ils sont encore très satisfaisants.

C'est une expérience du même genre que nous rapportons en prenant le poussin comme sujet et en utilisant le lait écrémé comme boisson.

Pour cela, sachant qu'un poussin boit deux fois plus qu'il ne mange, nous avons constitué des régimes de telle sorte que l'ensemble pâtée plus lait écrémé fournisse $\mathrm{I} 7,5 \mathrm{~kg}$ de protéines brutes par $100 \mathrm{~kg}$ de régime. Tous nos résultats sont rapportés à ceux du lot témoin (lot $\mathrm{X}$ ) nourri d'une pâtée commerciale classique dosant 2I p. Ioo de protéines. 


\section{PROTOCOLE EXPÉRIMENTAL}

Nous avons constitué au départ 8 lots de 25 poussins d'un jour, mâles, issus de croisements Sussex $\times$ Rhode Wyandotte. L'expérience a été faite en batterie d'élevage et a duré II semaines.

Les 7 lots expérimentaux se groupent en deux séries. Dans la première, lès poussins sont nourris dès le début de l'expérience avec les régimes à tester. Les poussins de la $z^{\mathrm{e}}$ série reçoivent l'aliment commercial de référence pendant les 5 premières semaines. Pratiquement, ce temps correspond au passage dans la batterie chauffée ou sous l'éleveuse. A la suite de quoi, ils reçoivent le régime expérimental qui leur est distribué, comme régime d'engraissement ou de finition.

La répartition des lots est donc la suivante:

\begin{tabular}{|c|c|c|c|}
\hline Lots & Aliments & Boissont & Observations \\
\hline $\begin{array}{l}\text { Première série : } \\
\qquad \mathrm{A}\end{array}$ & $\begin{array}{r}\text { Céréales t complément salin et } \\
\text { vitaminique } \ldots \ldots \ldots \ldots \ldots \ldots\end{array}$ & lait écrémé & de $\circ$ à I I semaines \\
\hline B & $\begin{array}{l}\text { Céréales + alim. complémentaire } \\
+ \text { pommes de terre } 20 \% \ldots\end{array}$ & lait écrémé & de o ¿ i t semaines \\
\hline C & $\begin{array}{l}\text { Céréales + alim. complémentaire } \\
+ \text { - pommes de terre } 40 \% \ldots\end{array}$ & lait écrémé & de $\circ \mathfrak{a}$ I I semaines \\
\hline $\mathrm{D}$ & $\begin{array}{l}\text { Céréales }+ \text { alim, complémentaire } \\
+ \text { ponmes de terre } 60 \% \ldots \ldots\end{array}$ & lait écrémé & de o à I I semaines \\
\hline $\begin{array}{l}\text { Deuxième série : } \\
\qquad \mathrm{E}\end{array}$ & $\begin{array}{l}\text { Aliment de référence } \ldots \ldots \ldots \ldots \\
\text { Céréales }+ \text { alim. complémentaire } \\
\quad+\text { pommes de terre } 30 \% \ldots \ldots\end{array}$ & lait écrémé & $\left\{\begin{array}{l}\text { de o di } 5 \text { semaines } \\
\text { de } 5 \text { à I I semaines }\end{array}\right.$ \\
\hline $\mathrm{F}$ & $\begin{array}{l}\text { Aliment de référence ........... } \\
\text { Céréales + pommes de terre } 30 \%\end{array}$ & lait écrémé & $\begin{array}{l}\text { de } 0 \text { à } 5 \text { semaines } \\
\text { de } 5 \text { à ix semaines }\end{array}$ \\
\hline$G$ & $\begin{array}{l}\text { Aliment de référence ............ } \\
\text { Céréales scules .............. }\end{array}$ & $\begin{array}{l}\text { eau } \\
\text { lait écrémé }\end{array}$ & $\begin{array}{l}\text { de o à } 5 \text { semaines } \\
\text { de } 5 \text { à I I semaines }\end{array}$ \\
\hline
\end{tabular}

I es céréales, le lait écrémé et les pommes de terre forment la majeure partie de ces rations expérimentales. Toutefois, il est bien évident qu'tun tel régime, richement pourvu en pommes de terre (certaines rations en renferment jusqu'à $60 \mathrm{p}$. Ioo) ne peut titrer $17,5 \mathrm{p}$. Ioo de protéines. Pour y parvenir nous avons fait entrer dans la ration un aliment complémentaire.

I a composition des régimes est donnée dans les tableaux I et II.

I es pommes de terre sont cuites chaque matin à l'autoclave pendant six minutes pour rendre la fécule plus digestible. Elles sont mélangées ensuite au reste de la ration en proportions convenables.

Le lait écrémé non coupé d'eau est distribué chaque jour. Régimes et lait sont fournis à volonté aux poussins pendant la journée.

Un soin tout particulier est apporté à la préparation des repas et on attache principalement beaucoup de prix à la propreté des mangeoires 
(IV, I958)

L'ALIMENTATION DU POUSSIN

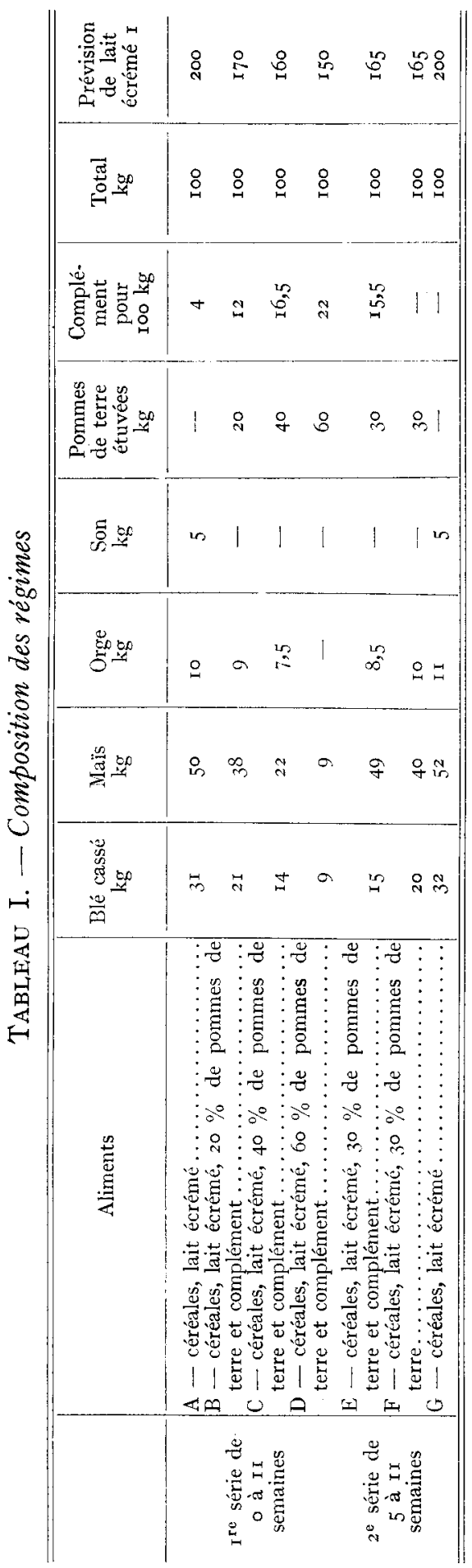

\begin{tabular}{|c|c|c|}
\hline 8ิ диәшоху әр әи!ाв & $\begin{array}{ll}2 \\
0 \\
0\end{array} \mid 11$ & 111 \\
\hline 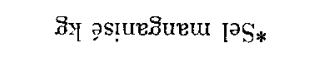 & 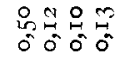 & $\underset{\substack{2 \\
0 \\
0}}{0} \mid$ \\
\hline Siy so،p әxpnod & 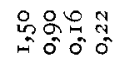 & $\begin{array}{ll}2 \\
0\end{array} \mid$ \\
\hline Sуч ипюте әр әұвиочівว & 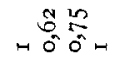 & \begin{tabular}{c|c}
$\infty$ \\
$\infty$ \\
0 \\
0
\end{tabular} \mid 1 \\
\hline 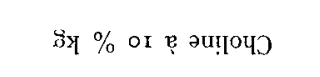 & $\begin{array}{l}1=00 \\
1=00 \\
0=0\end{array}$ & 虽| \\
\hline 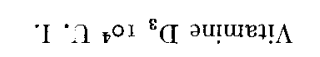 & $\operatorname{rog} \pi \hat{0}$ & $\stackrel{0}{q} \mid 1$ \\
\hline 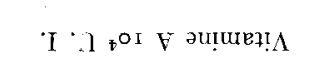 & 우율 & $\div 11$ \\
\hline מ̆. әихәzп әр әи!. & 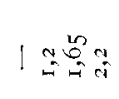 & 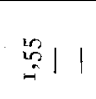 \\
\hline$\delta y$ & 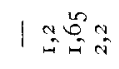 & $\begin{array}{ll}12 \\
2 \\
2\end{array}$ \\
\hline В̆Ү uosstod әр әи!л: & $\begin{array}{l}1020 \\
=90\end{array}$ & $\begin{array}{lll}2 & 0 \\
0 & 1 & 1\end{array}$ \\
\hline 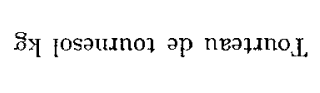 & 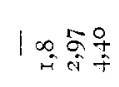 & 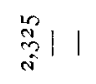 \\
\hline Sy $\operatorname{clos}$ әр пвәдxnoL & 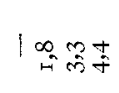 & $\begin{array}{ll}\infty & 0 \\
i & 1 \\
i & 1\end{array}$ \\
\hline 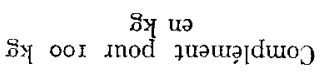 & 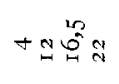 & 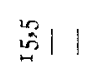 \\
\hline \multirow[t]{2}{*}{ 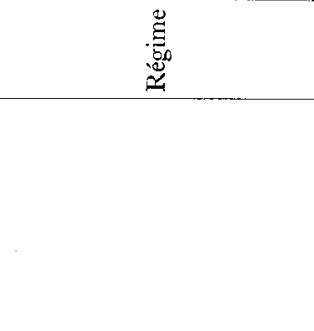 } & $4 D 00$ & 电地o \\
\hline & 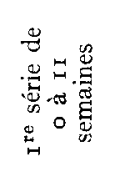 & 总 \\
\hline
\end{tabular}


et des abreuvoirs. Ces récipients sont retirés le soir, trempent dans un bain détersif pendant la nuit et sont abondamment rincés pour éliminer toute trace de produits de fermentation.

I es poussins sont éclairés en lumière artificielle ro $h$ par jour.

La croissance est appréciée par des pesées hebdomadaires et individuelles des animaux. D'autres mesures portent sur les quantités de nourriture ingérée et de lait bu.

\section{RÉSULTATS}

Dans tous les lots, les animaux se portent bien. En aucun cas, il n'a été remarqué de troubles digestifs ou de diarrhées, ce qui laisse à entendre que le lait écrémé comme seule boisson n'est pas nuisible au poulet, même au poussin du premier âge. Bien au contraire, nos animaux sem. blent apprécier tout particulièrement cette boisson. Des calculs qui seront présentés ultérieurement confirment cette remarque.

L'aspect des animaux est normal ; les poulets en général sont bien emplumés. Toutefois, nous remarquons dans le lot $\mathrm{A}$, du picage fréquent et un énervement important peu favorables à une bonne croissance.

Les résultats portant sur le gain de poids des animaux de la série I sont les suivants :

TABLEAU III

Gains de poids des Poulets à 5 semaines et $\grave{a}$ II semaines.

\begin{tabular}{|c|c|c|c|c|}
\hline \multirow{3}{*}{ Jots } & \multicolumn{4}{|c|}{ Gain de joids moyen } \\
\hline & \multicolumn{2}{|c|}{$\Lambda_{5}$ semaines } & \multicolumn{2}{|c|}{ A I I semaines } \\
\hline & $\operatorname{crl} \mathrm{g}$ & en $\%$ de $\mathrm{X}$ & en $\mathrm{g}$ & en $\%$ de $X$ \\
\hline $\begin{array}{l}A-\text { Làit écrémé }+ \text { céré } \\
\text { ales } \ldots \ldots \ldots \ldots \ldots \ldots\end{array}$ & $301 \div 15,5$ & 76,5 & I $212: 87, \mathrm{I}$ & 77 \\
\hline $\begin{array}{l}\mathrm{B} \text { - Iait écrémé }+ \text { céré- } \\
\text { ales }+\mathrm{P} \text {. de } \mathrm{T} .20 \% \text {. }\end{array}$ & $335+20.1$ & 85 & $1377+50,6$ & 88 \\
\hline $\begin{array}{l}\text { C - Lait écrémé céré- } \\
\text { ales }+\mathrm{P} \text {. de } \mathrm{T} \text {. } 10 \% \text {. }\end{array}$ & $418 \pm 20,2$ & 106 & r $535 \pm 6$ r, 2 & 98 \\
\hline $\begin{array}{l}\mathrm{D-Lait} \text { écrémé }+ \text { céré- } \\
\text { ales }+\mathrm{P} \text {. de } \mathbf{T} \text {. 60\% } \%\end{array}$ & $45^{2}-22,7$ & I 15 & $435 \cdots 76,6$ & 93 \\
\hline $\begin{array}{l}\mathrm{X} \text { - eau }+ \text { régime témoin } \\
\text { du commerce } . . . . . . . .\end{array}$ & $394: 25,3$ & I00 & I $57 \mathrm{I} \div 67,6$ & 100 \\
\hline
\end{tabular}

A 5 semaines toutes les moyennes des gains de poids sont significativement différentes. A II semaines les différences entre les lots sont moins spectaculaires et en particulier il n'y a pas de différences significatives entre $\mathrm{C}, \mathrm{D}$ et $\mathrm{X}$.

Il apparaît de ces simples chiffres que l'éleveur peut obtenir un résul- 
tat très satisfaisant en fabriquant lui-même ses rations, avec l'aide d'un aliment concentré. Nous irons même plus loin puisqu'à 5 semaines les animaux qui reçoivent des pommes de terre aux doses de 40 à $60 \mathrm{p}$. Ioo ont une croissance supérieure aux animaux recevant le régime standard.

I,es lots groupés dans la deuxième série - pendant la période qui s'écoule de 5 à II semaines - sont plus nombreux que ceux de la première série. On a ajouté en effet à ces derniers trois lots $\mathrm{E}, \mathrm{F}$ et $\mathrm{G}$ où varient d'une part le taux de pommes de terre et d'autre part la présence ou l'absence d'aliment complémentaire. Le tableau IV met en évidence la nécessité d'apporter un complément à ces rations.

\section{TABLEAU IV}

Gains de poids moyens des animaux entre 5 et I I semaines $\left(2^{\mathrm{e}}\right.$ série $)$.

\begin{tabular}{|c|c|c|}
\hline Jots & Gain de poids en $g$ & Observations \\
\hline A - Lait, céréales, complément. & 9 II 20,8 & \multirow{5}{*}{$\begin{array}{l}\text { Avec aliment } \\
\text { complémentaire }\end{array}$} \\
\hline $\begin{array}{l}\text { B - Lait, céréales, complément, } \\
\text { P. de T. } 20 \% \text {............. }\end{array}$ & $1042 \pm 38,2$ & \\
\hline $\begin{array}{c}\mathrm{C}-\text { Lait, céréales, complément, } \\
\text { P. de T. } 40 \% . \quad \ldots \ldots \ldots .\end{array}$ & III $7+45,2$ & \\
\hline $\begin{array}{r}\mathrm{D}-\text { Lait, céréales, complément } \\
\text { P. de T. } 60 \% \ldots \ldots \ldots\end{array}$ & $1003 \pm 53$ & \\
\hline $\begin{array}{r}\mathbf{E}-\text { Lait, céréales, complément } \\
\text { P. de T. } 30 \% \ldots \ldots \ldots \ldots\end{array}$ & $\pm 15+\ldots 61,6$ & \\
\hline $\begin{array}{l}\text { F - Lait, céréales, }+ \\
\text { P. de T. } 30 \% \ldots \ldots \ldots \ldots\end{array}$ & $469-35,6$ & \multirow{2}{*}{$\begin{array}{l}\text { Sans aliment } \\
\text { complémentaire }\end{array}$} \\
\hline G - Lait, céréales ........ & $+69 \therefore 3-1,2$ & \\
\hline $\mathrm{X}$ - Aliment commercial ... & $\operatorname{II} 77 \therefore 51,6$ & $\begin{array}{l}\text { Témoin commun } \\
\text { aux deux séries }\end{array}$ \\
\hline
\end{tabular}

L'analyse statistique montre qu'il n'y a pas de différence significative entre $\mathrm{C}, \mathrm{E}$ et $\mathrm{X}$.

Nous pouvons donc tirer une première conclusion :

A la condition $q u$ 'ils renferment un aliment concentré équilibré, certains régimes du type "fermier " peuvent permettre une croissance normale chez le poussin. Ainsi, des régimes contenant 30 à $40 \mathrm{p}$. Ioo de pommes de terre cuites sont aussi efficaces que le régime témoin de référence. La valeur alimentaire de telles rations est donc bien établie.

Par contre, dans les lots qui ne reçoivent pas d'aliment concentré, la croissance continue normalement au cours des $6^{\mathrm{e}}$ et $7^{\mathrm{e}}$ semaines, mais ensuite décline lentement. I,es animaux finissent par perdre du poids. Cela tient au fait que la ration manque de vitamines. En effet, le lait écrémé est pattvre en vitamines $\mathrm{A}$ et $\mathrm{D}_{3}$ qui sont des facteurs de croissance importants. La preuve en est que le lot A qui reçoit le même régime supplémenté en vitamines a une croissance assez bonne. 
Les résultats portant sur les taux d'ingestion sont les suivants :

$$
\text { TABleiau } V
$$

Taux d'ingestion et indice de consommation.

\begin{tabular}{|c|c|c|c|c|c|c|c|c|}
\hline & Lots & $\begin{array}{l}\text { Matière } \\
\text { sèche de } \\
\text { la pâtée } \\
\text { ingérée } g\end{array}$ & $\begin{array}{l}\text { Matière } \\
\text { séche du } \\
\text { lait en } g\end{array}$ & $\begin{array}{c}\text { Matière } \\
\text { sèche } \\
\text { totale } \\
\text { ingérée g }\end{array}$ & $\begin{array}{l}\text { Protéines } \\
\text { ingćrées } g\end{array}$ & $\begin{array}{l}\text { Gain de } \\
\text { poids :r }\end{array}$ & I. C. & $\begin{array}{l}\text { Gain } \\
\text { de poids } \\
\text { protéines } \\
\text { ingérées }\end{array}$ \\
\hline $\begin{array}{l}\text { de o à II } \\
\text { semaines }\end{array}$ & $\begin{array}{l}\mathrm{A} \\
\mathrm{B} \\
\mathrm{C} \\
\mathrm{D} \\
\mathrm{X}\end{array}$ & $\begin{array}{l}3023 \\
3504 \\
3594 \\
3579 \\
4556\end{array}$ & $\begin{array}{r}794 \\
599 \\
949 \\
\text { I I I 3 } \\
-\end{array}$ & $\begin{array}{l}3^{817} 7 \\
4103 \\
45^{28} \\
4692 \\
455^{2}\end{array}$ & $\begin{array}{r}530 \\
683 \\
935 \\
\text { II } 93 \\
\text { I038 }\end{array}$ & $\begin{array}{l}I \geq I 2 \\
I 377 \\
I 535 \\
I 4,35 \\
r 57 x\end{array}$ & $\begin{array}{l}3,15 \\
2,98 \\
2,95 \\
3,27 \\
2,90\end{array}$ & $\begin{array}{l}2,28 \\
2,01 \\
1,64 \\
1,20 \\
1,51\end{array}$ \\
\hline $\begin{array}{l}\text { de } 5 \text { da I I } \\
\text { semaines }\end{array}$ & $\begin{array}{l}\mathrm{A} \\
\mathrm{B} \\
\mathrm{C} \\
\mathrm{D} \\
\mathrm{E} \\
\mathrm{F} \\
\mathrm{G} \\
\mathrm{X}\end{array}$ & $\begin{array}{l}2436 \\
2923 \\
2854 \\
2877 \\
3043 \\
2519 \\
2028 \\
3778\end{array}$ & $\begin{array}{l}634 \\
495 \\
765 \\
974 \\
696 \\
426 \\
528 \\
-\end{array}$ & $\begin{array}{l}3070 \\
3418 \\
3619 \\
3851 \\
3739 \\
2945 \\
2556 \\
3778\end{array}$ & $\begin{array}{l}426 \\
565 \\
748 \\
981 \\
756 \\
343 \\
363 \\
860\end{array}$ & $\begin{array}{r}9 \text { I I } \\
\text { IO } 42 \\
\text { I I I } \\
\text { I00, } \\
\text { I } 554 \\
469 \\
469 \\
\text { I I } 77\end{array}$ & $\begin{array}{l}3,37 \\
3,28 \\
3,24 \\
3,84 \\
3,24 \\
6,28 \\
5,45 \\
3,21\end{array}$ & $\begin{array}{l}2,1,3 \\
\mathrm{I}, 84 \\
\mathrm{I}, 49 \\
\mathrm{I}, 02 \\
\mathrm{I}, 52 \\
\mathrm{I}, 36 \\
\mathrm{I}, 29 \\
\mathrm{I}, 36\end{array}$ \\
\hline
\end{tabular}

Ces résultats appellent les observations suivantes :

\section{$I^{0}$ Poussins de la première série.}

En ce qui concerne les animaux de la première série (lots A B C I) X) observés de o à II semaines, les résultats sont dans l'ensemble assez satisfaisants. A II semaines, dans tous les cas sauf un, les poulets ont ingéré des quantités d'aliments, exprimées en matière sèche, égales ou inférieures à celle du lot de référence et les indices de consommation obtenus sont du même ordre de grandeur. On peut, à ce sujet, faire les remarques suivantes:

a) Au fur et à mesure que le taux de pommes de terre augmente dans le régime, on assiste à une élévation simultanée de la consommation totale d'aliments et de la quantité de protéines ingérées. La présence de pommes de terre cuites favorise l'appétit des poulets en dépit de la forte proportion d'eau qu'elles contiennent et de leur coefficient d'encombrement non négligeable. Toutefois, les pommes de terre présentent un certain inconvénient à être employées à trop fortes doses. C'est en effet pour le régime le plus riche en ce tubercule ( $60 \mathrm{p}$. Ioo) que l'indice de consommation est le moins bon et que l'intensité de la croissance cesse d'être maximale.

b) D'autre part, bien que la teneur en pommes de terre du régime conditionne l'appétit, elle est la cause de la réduction de l'efficacité protéique du régime; on peut aisément remarquer que la valeur du rapport 
$\frac{\text { gain de poids }}{\text { protéines ingérées }}$ va en s'abaissant quand le taux de pommes de terre augmente. Ceci est vraisemblablement en relation avec une diminution de l'utilisation digestive de l'aliment qui peut être expliquée par la richesse de ce tubercule en matières pectiques. Ces dernières sont principalement distribuées dans la pulpe (Vol,KSEN (6r)). Il en résulte d'ailleurs un abaissement du coefficient de digestibilité azotée de la pomme de terre puisque sa valeur n'est que de $63 \mathrm{p}$. roo chez le poussin ('Trrus (59)).

c) Il convient de souligner que le régime A composé d'un mélange de céréales comme pâtée et de lait écrémé comme boisson est celui qui fournit la plus haute efficacité protéique bien supérieure à celle du régime de référence (5I p. Ioo d'augmentation).

Nous apportons là une preuve de plus à la thèse selon laquelle les protéines du lait et des céréales se supplémentent parfaitement. Par contre, le fait que ce même régime ne permette qu'une croissance médiocre laisse à penser qu'une ration aussi simple ne fournit pas en suffisance les éléments nutritifs indispensables aux poulets.

\section{$2^{0}$ Poussins de la deuxième série}

Chez les animaux des lots A B C D observés de 5 à Ir semaines, nous retrouvons en gros les mêmes résultats que précédemment en ce qui regarde les quantités de matières sèches et de protéines ingérées d'une part et l'indice de consommation et l'efficacité protéique, d'autre part.

Parmi les lots appartenant en propre à la $2^{\mathrm{e}}$ série (lots $\mathrm{E}, \mathrm{F}$ et $\mathrm{G}$ ) les valeurs trouvées dans le cas du régime $\mathrm{E}$ (céréales, pommes de terre 30 p. Ioo, aliment complémentaire et lait écrémé) sont tout à fait comparables à celles fournies par le lot $\mathrm{C}$ qui reçoit dans les mêmes conditions 4o p. Ioo de pommes de terre. Par contre, dans le cas des régimes $\mathrm{F}$ et $\mathrm{G}$, qui ne contiennent pas d'aliments complémentaires et en particulier qui n'apportent pas de vitamines $\mathrm{A}$ et $\mathrm{D}$, la croissance est la plus mauvaise et les indices de consommation les plus élevés. Il ne semble donc pas possible de conduire correctement un élevage de poussins en usant comme seule source d'aliments d'un mélange de céréales, additionné ou non de pommes de terre, et de lait écrémé ; il nous paraît indispensable d'y adjoindre un aliment de complément pour pallier les carences d'un tel régime.

\section{$3^{\circ}$ Quantités de pâtée ingérée et de lait bu.}

Le tableau VI rassemble les valeurs du rapport de la quantité de lait bu à la quantité de pâtée sèche ingérée. Elles permettent de préciser comment la nature de la pâtée influence la consommation de la boisson.

On admet pratiquement que les poulets boivent 2 fois plus qu'ils 
ne mangent. En fait, nous constatons dans nos essais une élévation de cette valeur qui peut aller jusqu'à 3,3 sauf pour les lots B et F. Il est donc intéressant de noter que d'une part l'appétence du lait écrémé est grande et que d'autre part malgré une teneur en eau importante du régime, les rations contenant des pommes de terre favorisent, en général, l'ingestion de grandes quantités de boisson.

\section{TABLEAU VI}

Consommation relative de lait écrémé et de pâtée.

\begin{tabular}{|c|c|c|c|c|}
\hline & Lots & Jait bu $\mathrm{g}$ & $\begin{array}{l}\text { Matière sèche de } \\
\text { pâtée ingérée g }\end{array}$ & Rapport lait/pâtée \\
\hline de o à i I semaines & $\begin{array}{l}\mathrm{A} \\
\mathrm{B} \\
\mathrm{C} \\
\mathrm{D}\end{array}$ & $\begin{array}{r}8357 \\
6301 \\
9983 \\
\text { II } 708\end{array}$ & $\begin{array}{l}3023 \\
3504 \\
3594 \\
3579\end{array}$ & $\begin{array}{l}2,76 \\
1,80 \\
2,77 \\
3,27\end{array}$ \\
\hline de 5 à II semaines & $\begin{array}{l}\mathrm{A} \\
\mathrm{B} \\
\mathrm{C} \\
\mathrm{D} \\
\mathrm{E} \\
\mathrm{F} \\
\mathrm{G}\end{array}$ & $\begin{array}{r}6669 \\
5207 \\
8048 \\
10246 \\
7322 \\
4481 \\
5554\end{array}$ & $\begin{array}{l}2436 \\
2923 \\
2854 \\
2877 \\
3043 \\
2519 \\
2028\end{array}$ & $\begin{array}{l}2,73 \\
\mathbf{I}, 78 \\
2,82 \\
3,56 \\
2,40 \\
\mathbf{I}, 78 \\
2,73\end{array}$ \\
\hline
\end{tabular}

\section{$4^{\circ}$ Discussion de la méthode d'élevage.}

Il est intéressant pour l'éleveur de connaître non seulement l'efficacité pour la croissance des aliments étudiés, mais encore les performances qu'il peut espérer de ses élèves en fonction à la fois du régime et de la méthode d'élevage qu'il a suivis. C'est pourquoi, nous allons comparer nos résultats non plus sur la base du gain de poids mais au moyen du poids final que les Poulets ont atteint à 1'âge d'être vendus. Ces résultats sont résumés dans le tableau VII.

\section{TABLEAU VII}

Poids des animaux en grammes.

\begin{tabular}{|c|c|c|}
\hline Lots & A 5 semaines & A II semaines \\
\hline 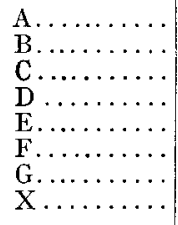 & $\begin{array}{l}342 \\
381 \\
463 \\
493 \\
433 \\
433 \\
433 \\
433\end{array}$ & $\begin{aligned} \text { I } 253 \\
\text { I } 4 \text { I } \\
\text { I } 591 \\
\text { I } 499 \\
\text { I } 587 \\
902 \\
902 \\
\text { I } 600\end{aligned}$ \\
\hline
\end{tabular}

Jusqu'à la cinquième semaine, la distribution des lots $\mathrm{E} F \mathrm{G}$ n'est pas effectuée. Les animaux qui leur sont destinés se trouvent réunis avec ceux du lot de référence et reçoivent le même régime. C'est pourquoi 
le poids moyen des poussins des groupes $\mathrm{E} F \mathrm{~F}$ et $\mathrm{X}$ sont identiques à 5 semaines. Notons qu'à cette date les poids moyens des lots $\mathrm{C}$ et $\mathrm{D}$ sont nettement supérieurs à celui du lot témoin. A l'âge commercial de la vente, les poids des poulets des lots $\mathrm{C}, \mathrm{E}$ et $\mathrm{X}$ sont tout à fait comparables. On peut donc en tirer deux conclusions :

- A la condition que le régime contienne un aliment de complément, et que le taux de pommes de terre ne dépasse pas $40 \mathrm{p}$. Ioo, il est possible d'obtenir avec une telle ration des poulets de poids comparables à ceux nourris d'un aliment complet.

- De plus, le mode d'élevage ne semble pas intervenir sur le poids final des animaux. Avec des rations de compositions très voisines ( $\mathrm{C}$ et $\mathrm{E}$ ) les résultats à II semaines sont exactement identiques. Il est donc possible d'élever des poussins dès le premier jour avec ces régimes.

Toutefois, il faut attirer l'attention sur le fait que n'importe lequel des régimes étudiés n'est pas pour autant satisfaisant, la preuve en est fournie par les lots $\mathrm{A}, \mathrm{F}$ et $\mathrm{G}$ pour lesquels l'aliment ne permet pas une croissance normale.

\section{CONCLUSION}

D'après ces premiers résultats, il semble donc que l'éleveur puisse non seulement nourrir ses poussins dès le premier âge avec les produits de son exploitation, mais encore que les régimes ainsi préparés soient parfaitement adaptés à ses préoccupations.

L'utilisation du lait comme boisson ne présente pas de contreindications dans l'alimentation du poussin à la condition expresse d'être récolté et distribué dans des récipients très propres.

Le régime constitué de plusieurs céréales et de lait écrémé, enrichi en vitamines $A$ et $D$, est un aliment protéique de haute valeur puisqu'il a fourni, dans nos essais, l'efficacité protéique la plus forte. Toutefois, pour obtenir ce résultat, la présence de minéraux et des vitamines $A$ et $D$ dans la ration est indispensable. Il convient cependant de répéter que, en dépit de ses qualités protéiques cet aliment ne présente pas toutes les garanties nutritionnelles puisqu'il ne permet pas une intensité de croissance optimale.

L'emploi de pommes de terre cuites dans le régime oblige du fait de sa faible teneur en protéines, à employer un aliment complémentaire. On remarque alors que plus le taux de pommes de terre augmente dans le régime, plus la consommation totale d'aliment augmente et par là même plus la quantité de protéines ingérées est élevée. Ceci entraîne une élévation parallèle dı gain de poids tant que le taux de ce tubercule n'est pas trop élevé.

Il semble que de tels régimes lorsqu'ils sont judicieusement consti- 
tués, puissent être utilisés pour 1'alimentation du poussin dès le premier jour. On peut également fournir un aliment complet du commerce pendant les premières semaines et réserver ces régimes " fermiers " comme aliment de finition.

En résumé, l'éleveur de volailles peut sans inconvénient établir les rations de ses poussins avec les produits récoltés sur son exploitation agricole : blé, orge, mais broyés, pommes de terre cuites, lait écrémé comme boisson, à la condition d'incorporer à la pâtée un aliment complémentaire de bonne qualité. Pour réussir, l'éleveur doit respecter les proportions dans lesquelles les différents aliments entrent dans la composition du régime et observer des règles très strictes quant à la propreté du matériel d'élevage.

Notre étude indique que les meilleturs résultats sont obtenus avec un régime constitué de 45 à 55 p. Ioo d'un mélange de grains, de 30 à 40 p. Ioo de pommes de terre cuites et de 15 p. Ioo d'un aliment complémentaire dosant 40 p. Ioo de protéines.

\section{Reçu pour publication le I4 mars 1957.}

\section{RÉFÉRENCES BIBLIOGRAPHIQUES}

(I) AbDerhalden (E.). - Z. Phys. Chem., 26, 498-500, I899.

(2) Atkinson (R. L.), Ferguson (T. M.) and Couch (J. R.). - Poul. Sci., 34, 855-86x, I955.

(3) Black (D. J. G.), Ge'TtY (J.), Morris (T. R.) and Pal.grave (J.). - J. Sci. Food. Agric., 3, 552-555, I952.

(4) Brock (R. J.) and Mitcheis, (H. H.). - Nutr. Abst. Reviews, 16, 249-278, I946.

(5) Booher (L. E.), Behan (I.) and McMeans (E.). - J. Nutr., 45, 75-IOO, I95I.

(6) Bourdet (A.). - Ann. Techn. Agric., 2, I8I-3I8, I956.

$(6$ bis) Bunge (A. H.) and AbDerhaldeen (E.). - Chem. Food and Nutr., $7^{\mathrm{e}}$ éd. Mc Millan Comp. N. Y., I vol., I950.

(7) Camp (A. A.), Cartrite (H. T.), Quisenkerry (J. H.) and Couch (J. R.). - Poul. Sci., 34, 559-566, I955.

(8) Castle (M. E.), Glucksmand (E. J.). - Dairy Industr., 13, 8, 767-769, I949.

(9) Chen (C. Y.) and Wang (I. C.). - Nutr. Bull. China, 1, I9-22, I94I.

(IO) CHICK (H.), SLACK (E. B.). - Biochem. J., 45, 2II-22I, I949.

(II) DEBRIT (F. P.). - Internat. Ztscht. Vitaminforsch, 24, 33I-345, I952.

(I2) Deshusses (J.) und Terrier. - Mitt. Geb. Lebensmittel Hyg., 31, 259-264, I940.

(I3) Dickens (F. L.), Parkhurst (R. T.) and Fellers (C. R.). - Poul. Sci., 20, 536-542, I 94 I.

(I4) Duncan (D. I.). - Nutr. Abstr. and $R$ ev., 25, 309-320, I955.

(I5) Fauchère (A.). - Ann. Nutr. Alim., 5, I87-230, I95I.

(I6) Fournier (P.), Dupuis (X.), Susbielile (H.) et Bourdeau (A.) - $-C . R$. Soc. Biol., 148, 265-268, I954.

(I7) Gerry (R. W.), Smyth (J. R.) and Howes (C. E.). - Poul. Sci., 30, 9r3, I95I. 
(I8) Hamilton (T. S.) and Card (L. E.). - J. Agr. Research, 27, 597-604, I924.

(I9) HART (E. B.). - Nutr. Rev., 10, I29-I30, I952.

(20) Hassinen (J. B.), Durbin (G. T.) and Bernhart (F. W.). - J. Nutr, 53, 249-257, I954.

(21) Heuser (G. F.), Scott (M. L.), Eskew (R. K.) and Edowards (P. W.). Poul. Sci., 30, 672-678, I95I. Xe Congrès Int. Avic. Edimbourg, Io2I04, I954.

(22) Höry (J.) und SANDVIK (Ø.). -- Nut. Abst. and Rev., 25, 580, I955.

(23) Jacouot (R.) et Armand (Y.). - C. R. Acad. Agric., 28, 595, I942.

(24) Jacquot (R.) et Trimbach (H.). - Bull. Soc. Sc. Hyg. Alim., 22, I-23, I934.

(25) Kon (S. K.). - Ann. Nutr. Alim., 5, I47-I63, I95I.

(26) Kon (S. K.) and Henry (K. M.). -J. Dairy Research, 16, 68-127, I949.

(27) Kon (S. K.) and Porter (J. W. G.). - Nutr. Abst. and Rev., 17, 3I-37, I $947-48$.

(28) Lane (R. I.), Johnson (E.) and Williams (R. R.). - J. Nutr., 23, 6I3$624, \mathrm{I} 942$.

(29) LEHMANN (F.). - Ztschr. Schweinezucht, 48, 89-9I, I94I.

(30) Leichsenring (J. M.), Norris (L. M.) and Pilcher (H. L.). - Food. Res., 22, 37-43, I957.

(3I) Leichsenring (J. M.), Pilcher (H. L.), Norris (L. M.). - Food. Res., 22, 44-50, I957.

(32) LEROy (A. M.), ZELTER (S.), et LERy (G.). - Ann. Zootechn., 1, 6I-78, I952.

(33) Leroy (A. M.), Zeiter (S.) et FeVrier (R.). - Ann. Zootechn., 1, 87-I46, I952.

(34) LeVy (J.) et Jacouot (R.). - C. R. Acad. Sci., 22\%, 37I-373, I948.

(35) Lilime (R. J.), BiRd (H. R.), SizemoRE (J. R.), KELI.OGG (W. L.) and DENTON (C. A.). - Poul. Sci., 33, 686-69I, I954.

(36) Menge (H.), Combs (G. F.), Hsu (P. T.) and Shorb (M. S.). - Poul. Sci., 31, 237-247, I952.

(37) McMIllan (A. M.) und Dudley (F. J.). - Harper Utility Poultry Journal, 25, I77-I78, I939-I940.

(38) Mitchel (H. H.) and Block (R. J.). - J. Biol. Chem., 163, 599-618, I946.

(39) Mormoto (H.) and Yoshida (M.). - Bull. Nat. Inst. Agric. Sci. Japan, 8, 43-5I, I954.

(40) Mulder (E. G.) and Bakema (K.). - Plant. and Soil., r, I35-I66, I956.

(4I) Mulier (R.) und Massberg (J.). - Arch. Tiernahrung, 4, 24I-257, I954.

(42) Ott (W. H.) and Boucher (R. V.). - Poul. Sci., 23, 497, 1944.

(43) Randoin (L.), Jourdan (C.). - C. R. Acad. Agric., 38, IoI-Io3, I952.

(44) Randoin (L.), Le Galime et Causeret (J.). - Table de composition des aliments, 8\%, $\mathrm{x} 947$.

(45) Riche'c (C.). - Presse Univ. (Paris), I vol, p. I6o., I949.

(46) Rochaix (A.) et TAPERNoux (A.). - Vigot ed. (Paris), I vol., 456 p., I 948.

(47) Roine (P.), Wichmann (K.) and Vihavainen (L.). - Acta. Agral. fenn., $83,7 \mathrm{I}-87$, I955.

(48) Rututer (W. J.), Wrichevsky (P.), Scot' (H. M.) and Hansen (R. G.). - Poul. Sci., 32, 706-7I5, I953.

(49) Schlamb (K. F.) and Bryant (R. L.). - Bull. N. Dakota Agric. Exp. Sta., 13, I04-I06, I95I.

(50) ScotT (M. L.). - Poul. Sci., 31, I75-I76, I952.

(5I) Shaw (T. P.). - Amer. J. Physiol., 31, 439-446, I9r3.

(52) Smonet (H.). — Bul. Soc. Sci. Hyg. Alim., p. 5I, I943.

(53) Spisni (D.). - Bol. Soc. Ital. Biol. Sper., 30, 66I-662, I954.

(54) Stevens (A. H.). - Nation. Butter Cheese J., 40, 28-29 et 6o-6r, I949.

(55) Szczyglowa (M.) et Siczkowna (J.). - Roczn. Panstwowego. Zokl. Hig., 3, I29-I44, I952. 
(56) Temperton (H.) and Dudiey (F. J.). - Harper Adams Poultry Journal (July I942).

(57) Temperton (H.) and Dudley (F. J.). - Jour. Agr. Sci., 33, 204-206, I943.

(58) Terroine (E. F.). - C. N. R. S., éd. I933.

(59) Titus (H. W.). - The Interstate ed. (Dauville Illinois), I vol., 253 p., I953.

(6o) Tré́molières (J.), Serville (Y.) et Jacquot (R.). - Soc. Française ed., 3 vol., I955.

(6I) Vouksen (N.). - Ztschr. Lebensmittel. Unterzuch. Forsch., 90, I77-I79, I950. 